\title{
Concurrent Ruptured Ectopic Pregnancy and Appendicitis
}

\author{
Nguyen Hien, MD, Khanh Le, MD, Connie Le, MD, and Hanh Nguyen, BS
}

The acute abdomen in pregnancy is a surgical emergency requiring timely diagnosis and therapy to avoid potentially high maternal and fetal morbidity and mortality. Ectopic pregnancy and appendicitis are 2 causes of acute abdomen in pregnancy. We present a case of concurrent ruptured ectopic pregnancy and appendicitis, and we review possible mechanisms that may underlie the pathogenesis of the simultaneous existence of these conditions in a patient.

\section{Case Report}

A 30-year-old woman, gravida 3, para 2, presented to the emergency department with a 2-day history of dull, bilateral, lower quadrant abdominal pain radiating to the back. The patient had experienced vaginal spotting for 3 days and heavy bleeding for 1 day. The last reported menstrual period was 6 weeks before presentation. She denied fevers, chills, vomiting, constipation, and diarrhea. Past medical, obstetric, gynecological, and surgical histories were remarkable only for 2 uncomplicated live births, and no history of sexually transmitted diseases or pelvic inflammatory disease.

The patient's vital signs included a temperature of $98^{\circ} \mathrm{F}$, blood pressure of $110 / 60 \mathrm{~mm} \mathrm{Hg}$, pulse of 85 beats/min, and respiratory rate of 14 breaths/ min. Physical examination revealed a closed cervix, no cervical notion tenderness, and mild right adnexal tenderness. Serum level of $\beta$-human chorionic gonadotropin ( $\beta$ hCG) was 20,000 IU/mL, and complete blood cell count revealed 8.6 leukocytes/ $\mathrm{mm}^{3}$, hematocrit of 0.33 , and platelet count of 220,000 . A transvaginal sonogram revealed echo-

Submitted, revised, 29 October 2004.

From the Department of Family Practice, George Washington University, Washington DC (NH, CL), the Department of Family Practice, Howard University Hospital, Washington DC (KL), and Virginia Commonwealth University, School of Medicine, Richmond (HN). Address correspondence to Dr. Nguyen Hien, 3808 Daniel's Run Court, Fairfax, VA 22030 (e-mail: htnguyen@gwu.edu). genic material in the uterine cavity consistent with possible retained products of conception and adnexa within normal limits. The findings were discussed with a radiologist, who stated that the appendix could not be visualized because of the surrounding echogenic material. In addition, the concerns about possible ectopic pregnancy were articulated with the radiologist, who noted no adnexal mass or free pelvic fluid suggestive of ectopic pregnancy. The initial impression was incomplete abortion, for which dilation and curettage was performed. The patient was discharged from the hospital with instructions to follow up immediately if she experienced fever, recurrent or worsening abdominal pain, vomiting, or other systemic complaints.

The patient returned to the emergency department 1 week later with persistent right lower quadrant abdominal pain that had worsened severely over the preceding 24 hours. There were associated fever, chills, vomiting, and anorexia, but no vaginal bleeding. The patient's vital signs included a temperature of $101.6^{\circ} \mathrm{F}$, blood pressure of $90 / 60 \mathrm{~mm}$ $\mathrm{Hg}$, heart rate of 108 beats/min, and respiratory rate of 20 breaths/min. The physical examination was significant for rebound tenderness in the right lower quadrant and was positive for McBurney point tenderness and psoas signs. Pelvic examination revealed closed cervix without cervical motion tenderness and moderate right adnexal fullness and tenderness. $\beta$ hCG was $500 \mathrm{IU} / \mathrm{mL}$, and complete blood cell count was significant for a leukocyte count of $18,0000 / \mathrm{mm}^{3}$ with $89 \%$ polymorphonuclear leukocytes and $10 \%$ bands, hematocrit of 0.30 , and a platelet count of 350,000. Cervical and urine cultures were negative for infection. Transvaginal sonogram revealed a right complex mass measuring $3 \times 4 \mathrm{~cm}$, minimal free fluid in the adnexa, and an empty sac. The findings were discussed with the radiologist, who stated that the appendix could not be well visualized. Preoperative 
diagnosis of acute abdomen was made, and the patient was taken emergently to laparotomy, which revealed a ruptured right fallopian tube, $500 \mathrm{~mL}$ of surrounding organized blood clot, adhesions extending from omentum to ascending colon, and a grossly inflamed appendix. Right salpingectomy, appendectomy, lyses of adhesions, and evacuation of blood clot were performed without complications.

In retrospect, sonographic findings at the initial visit were probably not accurately interpreted. The echogenic material visualized on the initial transvaginal sonogram was probably reactive decidua and hemorrhage rather than products of conception. Pathologic examination of the resected segment of appendix revealed edema and inflammation of the appendix without abscess formation, consistent with early appendicitis. Pathology of the resected right fallopian tube revealed immature placental tissue and villi consistent with ectopic pregnancy. Retrospective pathological review of the specimen from the initial dilation and curettage revealed decidua without chronic villi, which was not consistent with the earlier diagnosis of incomplete abortion. After surgery, the patient received intravenous cefazolin (Ancef). She was discharged from the hospital in stable condition 3 days later with a prescription for oral antibiotics.

\section{Discussion \\ Epidemiology}

Ectopic pregnancy has been rarely reported in conjunction with appendicitis; there are 22 such cases in the medical literature since $1960 .{ }^{1}$ We present a case in which a patient presented simultaneously with appendicitis and ipsilateral ruptured ectopic pregnancy. Abdominal pain that occurs during pregnancy is challenging to work up because of the broad range of differential diagnoses and distortions of anatomic relationships by the gravid uterus. Common gynecologic causes include ovarian torsion, degenerating leiomyomata, ruptured corpus luteum, pelvic inflammatory disease, and tubo-ovarian abscess. As the most common cause of surgical pain in pregnant patients, appendicitis is estimated to occur at an incidence of 1 per 1500 pregnancies. ${ }^{1}$ Ectopic pregnancies occur at a frequency of approximately 16 per 1000 patients. However, only 22 previous cases of simultaneous ectopic pregnancy and appendicitis have been doc- umented. Because of the potential for high maternal-fetal morbidity and mortality associated with either condition, the practitioner must be vigilant for these differential diagnoses in patients presenting with acute abdomen in pregnancy.

\section{Diagnosis}

The difficulty in diagnosing the acute abdomen in pregnancy is in attributable in part to the normal changes in physiologic and metabolic states and anatomic distortions in pregnant patients. ${ }^{1,2}$ The leukocyte count and sedimentation rate can be artificially elevated in the gravid state in the absence of infection. The appendix is characteristically pushed cephalad by the gravid uterus, so that typical signs of appendicitis, such as McBurney point tenderness and psoas signs, may not be elicited. ${ }^{1,3,4}$ Although advances in pelvic sonography by transvaginal and high frequency sonogram ${ }^{5}$ and highly sensitive tests for $\beta$ hCG have facilitated earlier diagnosis of ectopic pregnancy before the onset of clinical symptoms, differences in operator technique, and obscuring bowel and gas may render preoperative diagnosis of appendicitis and/or ectopic pregnancy inconclusive. A corollary of this is that lack of definitive findings on sonography (such as free pelvic fluid, echogenic adnexal mass for ectopic pregnancy, and noncompressible appendix $>6 \mathrm{~mm}$ with free fluid for appendicitis), in the presence of high clinical suspicion from a complete history and physical examination, should not preclude a differential diagnosis including appendicitis and ectopic pregnancy in the workup of acute abdomen in a pregnant patient. ${ }^{1-4}$

Our patient did not present with vaginal bleeding at the latter visit, when she was diagnosed with ruptured ectopic pregnancy. This demonstrates that ectopic pregnancy and appendicitis may not always present with classic historical, physical examination, laboratory, and radiographic features, particularly in pregnancy. Diagnoses of appendicitis and ectopic pregnancy were entertained preoperatively, but neither could be excluded by history, physical, laboratory, and radiology examinations. Because of the uncertainty in diagnosis, emergency exploratory laparotomy was pursued, leading to the surprising finding of concurrent ruptured ectopic pregnancy and appendicitis. Therefore, in patients with acute abdomen in pregnancy in whom there is diagnostic uncertainty, a surgical approach through 
laparoscopy or laparotomy can be invaluable for diagnosis and therapy. ${ }^{1,2,6}$

\section{Etiologic Inter-Relationships}

The risk of ectopic pregnancy increases with previous ectopic pregnancy and conditions leading to tubal damage and infertility such as pelvic inflammatory disease, prior tubal surgery, and smoking. ${ }^{7}$ It is interesting that in vitro fertilization for tubal infertility has been reported in conjunction with concurrent ectopic pregnancy and heterotopic pregnancy. ${ }^{2,6}$ Previous reports have described possible underlying etiologic inter-relationships between ectopic pregnancy and appendicitis. ${ }^{1,8,9}$ It is unknown whether appendicitis is coincidentally associated with ectopic pregnancy or is a possible risk factor for subsequent development of ectopic pregnancy. Some authors have contended that appendicitis or history of surgery for appendicitis may induce short- or longterm adjacent inflammation in the fallopian tubes that predisposes to subsequent development of ectopic pregnancy. ${ }^{1,7,8,9}$

On the other hand, some authors have contended that ectopic pregnancy may induce contiguous inflammation, leading to periappendicitis, then appendicitis. ${ }^{1,10}$ At present, there is no single unifying theory about the etiology of appendicitis, but a combination of luminal obstruction and/or infection is postulated as the most probable etiologic factor. The theory of luminal obstruction in the pathogenesis of appendicitis contends that lymphoid hyperplasia or fecaliths cause luminal obstruction. A subsequent increase in intraluminal pressure results in ischemia of the appendiceal wall. The infection theory in the pathogenesis of appendicitis contends that bacterial or viral enteric infections from processes such as gastroenteritis or colitis adjacent to the appendix initially lead to mucosal ulceration of the appendix and subsequent secondary infection from normal colonic flora. ${ }^{10}$

Consistent with the infection theory, we postulate that ectopic pregnancy may cause an initial contiguous inflammatory reaction in the adjacent appendix. This leads to mucosal sloughing and creates a portal for infection in the appendix by normal colonic bacterial flora. We postulate that ectopic pregnancy may trigger appendicitis through a combination of initial inflammation and secondary infection. Previously reported cases of concurrent ectopic pregnancy and appendicitis have indicated a predilection for right tubal ectopic pregnancy (75\%) versus left tubal ectopic pregnancy $(16 \%){ }^{1}$ These observations are consistent with the possibility for adjacent inflammation in right-sided ectopic pregnancy and appendicitis as a possible underlying pathogenic mechanism. Most of the cases above were characterized by the onset of symptoms in less than 1 week and the emergence of concurrent ectopic pregnancy and appendicitis. Our case of ruptured ectopic pregnancy differs from previously reported cases of ectopic pregnancy, most of which were unruptured. ${ }^{1}$

\section{Conclusion}

In conclusion, one must be vigilant for the possibility of simultaneous causes of abdominal pain in the workup of acute abdomen in a female patient, particularly during pregnancy when characteristic physiologic and anatomic relationships may be distorted. There may be mutual etiologic/pathogenic mechanisms that lead to concurrent development of both ectopic pregnancy and appendicitis through contiguous inflammatory and infectious processes. It is important that during operations for right tubal ectopic pregnancy, one must check the appendix to rule out concurrent appendicitis.

\section{References}

1. Riggs JC, Schiavello HJ, Fixler R. Concurrent appendicitis and ectopic pregnancy. A case report. J Reprod Med 2002;47:510-4.

2. Akman MA, Katz E, Damewood MD, Ramzy AI, Garcia JE. Perforated appendicitis and ectopic pregnancy following in-vitro fertilization. Hum Reprod 1995;10:3325-6.

3. Tracey M, Fletcher HS. Appendicitis in pregnancy. Am Surg 2000;66:555-9.

4. Andersen B, Nielsen TF. Appendicitis in pregnancy: diagnosis, management and complications. Acta Obstet Gynecol Scand 1999;78:758-62.

5. Molander P, Paavonen J, Sjoberg J, Savelli L, Cacciatore B. Transvaginal sonography in the diagnosis of acute appendicitis. Ultrasound Obstet Gynecol 2002;20:496-501.

6. Barnett A, Chipchase J, Hewitt J. Simultaneous rupturing heterotopic pregnancy and acute appendicitis in an in-vitro fertilization twin pregnancy. Hum Reprod 1999;14:850-1.

7. Spandorfer SD, Barnhart KT. Role of previous ectopic pregnancy in altering the presentation of sus- 
pected ectopic pregnancy. J Reprod Med 2003;48: $133-6$.

8. Bozoklu S, Bozoklu E, Ciftci A, Coskun T. Ruptured ectopic pregnancy with undetectable $\beta$-hCG levels coexisting with acute appendicitis. Acta Obstet Gynecol Scand 1997;76:181-2.

9. Chia P, Jeyarajah A. Co-existing tubal ectopic preg- nancy and appendicitis—a case report. Med J Malaysia 1996;51:485-7.

10. Feldman M, Friedman LS, Sleisenger MH, editors. Sleisenger \& Fordtran's gastrointestinal and liver disease: pathophysiology, diagnosis, management, 7th ed. Philadelphia: WB Saunders; 2002 . 